\title{
Concordancia diagnóstica de la evaluación de imágenes digitales clínicas y dermatoscópicas versus el examen clínico de lesiones tumorales de piel
}

\author{
Diagnostic concordance between clinical and dermatoscopic digital images' evaluation versus face to face \\ evaluation of skin tumors
}

\section{Catalina Santa-Vélez¹, Xavier Rueda², Leonardo Pulido², Carolina Pozzobón ${ }^{3}$ Ricardo Sánchez ${ }^{4}$, Álvaro Acosta ${ }^{5}$}

\footnotetext{
1. Médica dermatóloga, fellow de Dermatología Oncológica, Instituto Nacional de Cancerología-Universidad Militar Nueva Granada, Bogotá, D.C., Colombia

2. Médico dermatólogo oncólogo, Instituto Nacional de Cancerología, Bogotá, D.C., Colombia

3. Médica dermatóloga, especialista en Dermatoscopia Digital, Instituto Nacional de Cancerología, Bogotá, D.C., Colombia

4. Médico, asesor metodológico, Instituto Nacional de Cancerología; docente, Universidad Nacional de Colombia, Bogotá, D.C., Colombia

5. Médico dermatólogo oncólogo, Instituto Nacional de Cancerología; docente, Universidad Nacional de Colombia, Bogotá, D.C., Colombia
}

\section{INTRODUCCIÓN}

La Dermatología es una especialidad que puede ser prestada en el modelo de atención por telemedicina, ya que se basa en imágenes visuales fácilmente fotografiadas y se ha demostrado que dicho modelo ofrece ventajas para la valoración de pacientes en áreas remotas sin acceso al especialista. Cabe anotar que las fotografías deben tener ciertas especificaciones y estar acompañadas de datos clínicos de calidad.

\section{MÉTODOS}

Se llevó a cabo un estudio observacional de concordancia de pruebas diagnósticas. Dos dermatólogos practicaron independientemente el examen clínico a los pacientes que consultaron por lesiones tumorales al Instituto Nacional de Cancerología, e hicieron un diagnóstico teniendo en cuenta tanto la clínica como la dermatoscopia. Posteriormente, se tomaron fotografías clínicas y dermatoscópicas de cada lesión para su posterior evaluación independiente por parte de tres teledermatoscopistas. Uno de los tres teledermatoscopistas tiene una maestría en dermatoscopia a la cual le dedica la mayor parte de su tiempo, y los otros dos son dermatólogos oncólogos con 20 años de experiencia, aproximadamente.

\section{RESULTADOS}

Se incluyeron 114 lesiones tumorales y se encontró una muy buena concordancia para la clasificación de las lesiones en melanocíticas benignas, melanocíticas malignas, no melanocíticas benignas o no melanocíticas (índice kappa de 0,72). La

\section{Correspondencia:}

Catalina Santa-Vélez

\section{Email:}

catalinasantavelez@yahoo.com

Recibido: $07 / 11 / 17$

Aceptado: 28/01/18

\section{Conflictos de interés:}

No se reportan conflictos de interés.

\section{Financiación:}

toda la financiación del presente estudio se realizó por parte del Instituto Nacional de Cancerología. 
decisión sobre el tratamiento recomendado presentó una muy buena concordancia diagnóstica (índice kappa de o,63). La concordancia fue baja para el diagnóstico principal de las lesiones (índice kappa de o,19). La concordancia diagnóstica entre el diagnóstico de los cinco evaluadores (tanto presenciales como teleevaluadores) y el diagnóstico histopatológico, fue moderada (índice kappa entre 0,33 y 0,44).

\section{CONCLUSIONES}

Los hallazgos sugieren que la teledermatología puede ser una herramienta valiosa para hacer el triage de lesiones tumorales y, así, mejorar los tiempos de espera y la oportunidad en los casos más urgentes; asimismo, ayudaría a priorizar los pacientes con lesiones tumorales malignas de residentes en áreas remotas sin acceso al especialista.

PALABRAS CLAVE: telemedicina, dermatoscopia, triage, fotografía

\section{INTRODUCTION}

Dermatology is a very visual specialty that is suitable for telemedicine care since it is based on visual images easily photographed. The telemedicine care model offers advantages for the assessment of patients in remote areas without access to the specialist. Thus, we wanted to evaluate if it is a reliable modality for the diagnosis of tumor lesions in our geographical area.

\section{OBJECTIVE}

To evaluate the concordance between the diagnoses made with clinical and dermatoscopic digital images versus the face-to-face skin examination diagnosis of skin tumor lesions from patients who consulted the Instituto Nacional de Cancerología between September $1^{\text {st }}$ and December 31 $31^{\text {st }}, 2016$.

\section{METHODS}

We included patients who consulted the Servicio de Dermatología Oncológica of the Instituto Nacional de Cancerología with presumptively skin tumors who underwent clinical and dermatoscopic examination by two independent dermatologists. Clinical and dermatoscopic digital photographs were taken and subsequent evaluation by three independent teledermatoscopists who made a diagnosis based on digital images. The interobserver diagnostic concordance (non-weighted kappa coefficient) for diagnosis by digital and dermatoscopic digital images vs the diagnosis by face-to-face clinical examination was estimated.

\section{RESULTS}

One hundred and fourteen tumor lesions were included. A very good concordance was found for the classification of benign melanocytic, malignant melanocytic, benign or malignant non-melanocytic lesions between the on-site evaluators and the tele-evaluators (kappa index: 0.72). The agreement for the recommended therapeutic management of these lesions showed a very good diagnostic concordance between dermatologists and tele-evaluators (kappa index: 0.63). The concordance was low for the primary diagnosis of the lesions (kappa index: 0.19). The diagnostic concordance between the diagnosis by the five evaluators (both face-to-face and tele-evaluators) and the pathological diagnosis was moderate (kappa index: 0.33-0.44).

\section{CONCLUSIONS}

The findings suggest that teledermatology can be a valuable tool for the triage of tumor lesions and thus improve waiting times and opportunity in the most urgent cases in patients from remote areas that do not have access to specialized on-site care.

KEY WORDS: telemedicine, dermoscopy, triage, photography 


\section{INTRODUCCIÓN}

La conveniencia, el costo-efectividad y la facilidad de aplicación de la telemedicina con los avances tecnológicos actuales, la han convertido en una modalidad de atención cada vez más atractiva que puede ser sincrónica o asincrónica ${ }^{(1)}$. Se practica en muchos países del mundo y su aceptación por parte de los dermatólogos viene en aumento ${ }^{(2)}$. Una de sus principales ventajas es el acceso a la atención especializada en lugares remotos; sin embargo, llama la atención que la gran mayoría de publicaciones sobre el tema proviene de Europa y Norteamérica, mientras que otras áreas con pobre distribución geográfica de médicos especialistas están poco representadas en la literatura científica ${ }^{3}$. Teniendo en cuenta que en Colombia existen barreras de acceso para la atención en salud y que muchas veces los pacientes de áreas rurales llegan a la consulta especializada en estados avanzados de la enfermedad por la poca disponibilidad o acceso a la consulta de dermatología, se quiso hacer una investigación para evaluar el comportamiento de esta modalidad de atención en nuestro medio.

En varios estudios de teledermatología, se describe el papel del melanographer, una persona entrenada en registrar la historia clínica del paciente con énfasis en la lesión por la cual consulta y los factores de riesgo para el cáncer de piel, que puede ser un médico general o una enfermera ${ }^{(4,5)}$. Así, se planteó hacer un ejercicio similar registrando los datos en un formato diseñado para tal fin y, de esta forma, estarían disponibles para los teleevaluadores. Ya se ha demostrado en múltiples estudios que la teledermatoscopia mejora el rendimiento diagnóstico ${ }^{(6-9)}$, por lo que se decidió incluir fotografías de dermatoscopia para la teleevaluación. En la práctica, este modelo de atención puede ser factible y costo-efectivo; se describe en regiones como Australia y Nueva Zelanda (países con la más alta incidencia y prevalencia de cáncer de piel) ${ }^{(5)}$, donde se cuenta con médicos y enfermeras entrenados en teleconsulta y con un dermatoscopio de similares características a las del presente estudio.

En algunos estudios, la concordancia diagnóstica de la teledermatología frente a la evaluación presencial varía según el área geográfica, lo que puede deberse al fototipo de piel de los pacientes, la prevalencia de los distintos tipos de cáncer de piel, los patólogos y los aspectos médico-legales que pueden llevar a un menor umbral para el diagnóstico de melanoma por temor a posibles demandas ${ }^{(4)}$.

El objetivo del trabajo fue evaluar la concordancia entre el diagnóstico por imágenes digitales clínicas y derma- toscópicas frente al examen clínico, en lesiones tumorales de piel de pacientes que consultaron al Instituto Nacional de Cancerología entre el $1^{\circ}$ de septiembre y el 31 de diciembre de 2016.

\section{METOdOLOGÍA}

Se llevó a cabo un estudio observacional de concordancia de pruebas diagnósticas. Se incluyeron los pacientes mayores de 18 años de edad que consultaron por lesiones tumorales de piel al Servicio de Dermatología Oncológica del Instituto Nacional de Cancerología. Se obtuvo la firma del consentimiento informado, en el que se manifestaba su aceptación para el registro fotográfico. Se excluyeron los pacientes cuya lesión no pudiera ser fotografiada y solo fuera detectable al tacto.

Para seleccionar el número y el tipo de lesión se dio prioridad a las lesiones que el paciente señaló como más preocupantes, un máximo de tres lesiones por paciente. Este criterio se basa en que la aplicabilidad de la prueba sería una situación en la que el paciente acudiría a consulta por la presencia de la lesión. Con ello se buscaba atenuar el sesgo de selección.

\section{TAMAÑO DE LA MUESTRA}

Para proteger la validez estadística del estudio, se calculó previamente el tamaño de la muestra de manera que tuviera un poder de o,8 y se consideró validar probabilísticamente la prueba de concordancia entre las dos evaluaciones mediante el índice kappa.

Se calculó que se requería un tamaño de muestra de 114 lesiones, con lo que se lograría el poder del $80 \%$ para detectar un valor kappa de o,50 en una prueba bilateral de $\mathrm{H}_{\mathrm{o}}$ : kappa=0,30 Vs. $\mathrm{H}_{1}$ : kappa $\neq 0,30$, cuando hay tres categorías con frecuencias iguales a 0,30, 0,30 y 0.40 . Este cálculo del poder se basa en un nivel de significación de 0,05.

Se considera que la concordancia entre observadores es excelente y casi perfecta, cuando el índice kappa de Cohen es de o,81 a 1,00; substancial, si es de o,61 a o,80; moderada, si es de 0,41 a 0,60; aceptable, si es de 0,21 a 0,40; baja, si es de 0,o a 0,2, y mala, si es menor de ${ }^{(10)}$.

Los datos sociodemográficos de los pacientes (edad, sexo y ocupación), los factores de riesgo para cáncer de piel (fototipo, color de los ojos, color del pelo, historia personal o familiar de melanoma, presencia de nevos atípicos, daño actínico, inmunosupresión, fotoexposición e historia de quemaduras solares) e his- 
toria de la lesión (tiempo de aparición, localización y sintomatología asociada), se consignaron en un formato diseñado para tal fin. Se seleccionaron aquellas lesiones que el paciente hubiera señalado como más preocupantes.

Luego de definido el número y el tipo de lesiones, un dermatólogo -evaluador presencial- procedió a tomar la fotografía clínica de la lesión, con una cámara fotográfica digital semiprofesional marca Canon Powershot $^{\mathrm{TM}}$, modelo GLPH de 30oHS, 12,1 megapíxeles por $\mathrm{cm}^{2}$. La fotografía clínica se tomó ubicando el lente de la cámara a una distancia de $15 \mathrm{~cm}$ de la lesión, en proyección perpendicular (ángulo de $90^{\circ}$ ) al centro de la lesión, bajo condiciones estándar de luz natural (sin activar el flash de la cámara). Seguidamente, se tomó la fotografía dermatoscópica con el equipo Dermlite hybrid $^{\mathrm{TM}}$, modelo II y la misma cámara fotográfica, sin contacto para mejor visualización de las estructuras vasculares.

A continuación, los dos dermatólogos presenciales -teniendo en cuenta los datos sociodemográficos, la historia de la lesión y los factores de riesgo para cáncer de piel- de manera ciega e independiente, hicieron la evaluación clínica y dermatoscópica presencial y llenaron un formato diagnóstico.

Cada lesión se clasificó en una de las siguientes categorías: lesión melanocítica benigna, lesión melanocítica maligna, lesión no melanocítica benigna, lesión no melanocítica maligna. Luego hicieron un diagnóstico principal y un diagnóstico diferencial, escogiendo entre los siguientes: queratosis seborreica, lentigo solar, melanoma, melanoma in situ, nevo atípico, nevo benigno, carcinoma basocelular, carcinoma escamocelular, carcinoma escamocelular in situ, queratosis actínica, dermatofibroma, angioma u otro; esta última categoría incluía un grupo misceláneo de condiciones dermatológicas: psoriasis, condrodermatitis nodularis helicis, picaduras de insectos, quistes, granulomas, verrugas virales, hiperplasias sebáceas y neurofibroma.

Asimismo, cada uno indicó el manejo terapéutico que recomendaba para el caso específico: biopsia de incisión, biopsia de escisión, seguimiento, otro tratamiento (crioterapia, 5-fluorocilo, etc.) o ningún tratamiento.

Las fotografías digitales clínicas y dermatoscópicas de la lesión, al igual que el formato con los datos sociodemográficos, la historia de la lesión y los factores de riesgo del paciente, fueron evaluadas independientemente posteriormente por tres médicos dermatólogos que actuaron como teledermatoscopistas y diligenciaron el mismo formato de valoración diagnóstica clasificando la lesión, quienes hicieron un diagnóstico principal y uno diagnóstico diferencial, y recomendaron un tratamiento teniendo en cuenta las mismas variables mencionadas anteriormente. Ninguno de los evaluadores conocía el diagnóstico de los demás.

Una vez hecha la evaluación para el estudio, los pacientes continuaron su proceso de atención clínica a cargo de su médico especialista tratante, sin influencia en el cuidado estándar del paciente. Cuando a criterio clínico del médico dermatólogo tratante se requirió estudio histopatológico, este fue interpretado por un médico patólogo con experiencia en dermatopatología. El objetivo principal era evaluar la concordancia entre el diagnóstico presencial y el telediagnóstico; se hizo un análisis secundario para evaluar la concordancia del diagnóstico presencial y el diagnóstico histopatológico, y entre el telediagnóstico y el diagnóstico histopatológico en el subgrupo de pacientes a los que se les había practicado biopsia.

\section{CONSIDERACIONES ÉTICAS}

Este estudio se desarrolló de acuerdo con los considerandos de la Declaración de Helsinki, las recomendaciones nacionales consignadas en la Resolución 8430 de 1993 del Ministerio de Salud de Colombia y la Resolución 2378 de 2008 del Ministerio de la Protección Social. El estudio fue aprobado por el Comité de Ética del Instituto Nacional de Cancerología.

\section{ANÁLISIS ESTADÍSTICO}

Los datos se consignaron en una base de datos diseñada en el programa RedCap 7.1.2 ${ }^{\mathrm{TM}}$. Para garantizar la calidad de la base de datos, se efectuaron dos grabaciones independientes de todas las variables; dichas grabaciones fueron posteriormente comparadas y las inconsistencias se ajustaron con los documentos fuente. Posteriormente, se exportó la información validada al programa Stata $13^{\mathrm{TM}}$ para su análisis estadístico.

Se hizo un análisis descriptivo de las frecuencias y de los porcentajes para las variables discretas, y para las variables continuas, medidas de tendencia central (medias o medianas) junto con sus correspondientes medidas de dispersión (desviaciones estándar o rango intercuartílico, respectivamente). Para efectos de la evaluación del grado de concordancia entre los diferentes evaluadores, incluyendo el resultado de la histopatología, se efectuó un análisis de concordancia de pruebas diagnósticas, para lo cual se calculó el coeficiente kappa no ponderado de Cohen. 


\section{RESULTADOS}

Se incluyeron 38 pacientes y 114 lesiones tumorales de piel (tabla 1). Los pacientes tenían un promedio de edad avanzada (72 años) con una proporción similar de hombres (49\%) y mujeres (51 \%). La mayoría tenía como ocupación ama de casa o agricultor. En la muestra no hubo pacientes de raza negra y, tampoco, de poblaciones indígenas.

Los factores de riesgo para cáncer de piel se resumen en la tabla 2. La mayoría de los pacientes tenían piel de fototipo II; el 10,5\% tenía antecedentes personales de melanoma; el 2,6 \%, antecedentes familiares de melanoma; el $89 \%$, evidencia de daño actínico, y el $10 \%$, antecedentes de quemadura solar.

En la tabla 3 se presentan las variables descriptivas de las lesiones. El 66 \% de las lesiones se encontraba en cabeza o cuello; el tiempo promedio de evolución era de 13,2 \pm 21,2 meses, con un rango de 1 mes a 6,5 años. Se encontró que solo la cuarta parte de las lesiones presentaba sintomatología asociada.

Así, teniendo en cuenta la valoración dermatológica presencial como pruena de referencia, se encontró que los índices kappa para la concordancia de los diferentes diagnósticos nosológicos principales entre los cinco dermatólogos, fueron bajos (kappa combinado de o,19) (tabla 4). Sin embargo, en los índices kappa para la concordancia entre los cinco evaluadores para la clasificación de las lesiones se encontró una concordancia combinada muy buena, la cual fue mayor para las lesiones no melanocíticas malignas y las melanocíticas benignas; $y$ un poco menor pero igualmente muy buena, para las no melanocíticas benignas $(0,66)$ y las melanocíticas malignas (tabla 5). La concordancia entre los dermatólogos sobre la conducta terapéutica recomendada, fue muy buena (tabla 6).

Hubo diferencias no significativas entre la concordancia de los diagnósticos principales hechos por los dermatólogos y el diagnóstico histopatológico de las lesiones que tenían biopsia; fue mayor la concordancia para la dermatóloga con mayor experiencia en dermatoscopia, lo que refleja la importancia del grado de entrenamiento en la precisión diagnóstica (tabla 7).

Algunos ejemplos representativos de la discordancia entre el diagnóstico de los dermatólogos presenciales y el de los teleevaluadores, se presentan en la figura 1.

Tabla 1. Características demográficas: análisis descriptivo

\begin{tabular}{|c|c|}
\hline Variable (medida de resumen) & Resultado ${ }^{\ddagger}$ \\
\hline Edad (años), promedio (DE) $)^{\S}$ & $72,8(11,9)$; rango: $39-92$ \\
\hline Sexo femenino, n (\%) & $58(50,9)$ \\
\hline Ocupación, ama de casa, n (\%) & $39(34,2)$ \\
\hline Agricultor, n (\%) & $30(26,3)$ \\
\hline Oficios varios, $n$ (\%) & $15(13,1)$ \\
\hline Cesante, n (\%) & $12(10,5)$ \\
\hline Militar, n (\%) & $6(5,3)$ \\
\hline Minero, n (\%) & $3(2,6)$ \\
\hline Oficinista, n (\%) & $3(2,6)$ \\
\hline Comerciante, $\mathrm{n}(\%)$ & $3(2,6)$ \\
\hline Ganadero, n (\%) & $3(2,6)$ \\
\hline Raza blanca, n (\%) ${ }^{\ddagger}$ & $69(60,5)$ \\
\hline Raza mestiza, n (\%) & $45(39,5)$ \\
\hline
\end{tabular}


Tabla 2. Descripción de los factores de riesgo para cáncer de piel

\begin{tabular}{|c|c|}
\hline Variable & $\mathrm{n}(\%)^{\ddagger}$ \\
\hline Fototipo de piel, I & $30(26,3)$ \\
\hline II & $45(39,5)$ \\
\hline III & $33(29,0)$ \\
\hline IV & $6(5,26)$ \\
\hline Color del cabello, blanco & $24(21,1)$ \\
\hline Gris & $12(10,5)$ \\
\hline Pelirrojo & $3(2,6)$ \\
\hline Rubio & $24(21,1)$ \\
\hline Castaño & $36(31,6)$ \\
\hline Negro & $15(13,2)$ \\
\hline Color de los ojos, azules & $27(23,7)$ \\
\hline Verdes & $12(10,5)$ \\
\hline Castaños & $66(57,9)$ \\
\hline Negros & $9(7,8)$ \\
\hline Historia familiar de melanoma & $3(2,6)$ \\
\hline Historia personal de melanoma & $12(10,5)$ \\
\hline Historia de nevos atípicos & $6(5,3)$ \\
\hline Cambio en los lunares referido por el paciente, número & $105(92,1)$ \\
\hline (No sabe) & $9(7,9)$ \\
\hline Daño actínico & $102(89,5)$ \\
\hline Historia de quemaduras solares & $12(10,5)$ \\
\hline Exposición solar (actividades al aire libre de manera crónica) & $111(97,4)$ \\
\hline Inmunosupresión, (n) & $o(0,0)$ \\
\hline
\end{tabular}

₹ Todos los porcentajes se obtuvieron con referencia al total de la muestra

$(\mathrm{N}=114)$.

Tabla 3. Descripción de la evolución y las características macroscópicas de la lesión

\begin{tabular}{|c|c|}
\hline Variable (medida de resumen) & Resultado ${ }^{\ddagger}$ \\
\hline Tiempo de evolución (meses), promedio (DE) & $13,2(21,9)$ \\
\hline Presentación de síntomas, sí (\%) & $28(24,6)$ \\
\hline Localización, cabeza y cuello, n (\%) & $76(66,7)$ \\
\hline Tronco, n (\%) & $18(15,8)$ \\
\hline Miembros inferiores, $\mathrm{n}(\%)$ & $14(12,3)$ \\
\hline Miembros superiores, $\mathrm{n}(\%)$ & $5(4,4)$ \\
\hline Genitales & $1(0,9)$ \\
\hline Presentación de síntomas, sí, n (\%) & $28(24,6)$ \\
\hline No, $n(\%)$ & $86(75,6)$ \\
\hline Características referidas por el paciente, prurito, n (\%) & $13(11,4)$ \\
\hline Dolor o ardor, n (\%) & $12(10,5)$ \\
\hline Eritema, $\mathrm{n}(\%)$ & $0(0,0)$ \\
\hline Sangrado, n (\%) & $6(5,3)$ \\
\hline Cambio de color, n (\%) & $2(1,8)$ \\
\hline Crecimiento, $n(\%)$ & $5(4,39)$ \\
\hline Trauma, n (\%) & $1(0,9)$ \\
\hline Tamaño de la lesión $(\mathrm{cm})$, promedio & $0,99 \times 0,83$ \\
\hline Mediana, centímetros & $0,75 \times 0,70$ \\
\hline \multicolumn{2}{|c|}{$\begin{array}{l}\text { ‡ Todos los porcentajes se obtuvieron con referencia al total de la muestra } \\
(\mathrm{N}=114) \text {. }\end{array}$} \\
\hline
\end{tabular}


Tabla 4. Índices kappa para concordancia de los diagnósticos principales de las lesiones incluidas, hechos por los cinco dermatólogos

\begin{tabular}{lc} 
Diagnóstico & Kappa* \\
Queratosis seborreica & 0,12 \\
Lentigo solar & 0,246 \\
Melanoma & 0,075 \\
\hline Melanoma in situ & 0,073 \\
Nevo atípico & 0,063 \\
\hline Nevo benigno & 0,211 \\
Carcinoma basocelular & 0,073 \\
\hline Carcinoma escamocelular & 0,323 \\
Carcinoma escamocelular in situ & 0,276 \\
\hline Queratosis actínica & 0,1 \\
\hline Angioma & 0,003 \\
\hline Otro & 0,23 \\
\hline Kappa combinado & 0,194 \\
* Para todos los casos, pro,ooo1 &
\end{tabular}

Tabla 5. Índices kappa para concordancia diagnóstica de la clasificación de las lesiones hecha por los cinco dermatólogos

\begin{tabular}{lc} 
Clasificación de la lesión & Kappa* \\
Melanocítica benigna & 0,778 \\
Melanocítica maligna & 0,533 \\
\hline No melanocítica benigna & 0,662 \\
Melanocítica maligna & 0,806 \\
Índice kappa combinado & 0,725
\end{tabular}

* Para todos los casos, pro,0001

Tabla 6. Índices kappa para concordancia diagnóstica de la conducta terapéutica para las lesiones incluidas, recomendada por los cinco dermatólogos

$\begin{array}{lc}\text { Conducta terapéutica recomendada } & \text { Kappa* } \\ \text { Biopsia } & 0,652 \\ \text { Seguimiento sin ningún tratamiento } & 0,652 \\ \text { Otro tratamiento (crioterapia, 5-FU, etc.) } & 0,481 \\ \text { Índice kappa combinado } & 0,634 \\ \text { * Para todos los casos, p<o,0001 } & \end{array}$

Tabla 7. Concordancia entre el diagnóstico de cada dermatólogo y el diagnóstico histopatológico de las lesiones con biopsia

\begin{tabular}{lc} 
Dermatólogo evaluador & Kappa* \\
Presencial 1 & 0,368 \\
\hline Presencial 2 & 0,332 \\
Teleevaluador 1 & 0,391 \\
\hline Teleevaluador 2 & 0,374 \\
Teleevaluador 3 & 0,444
\end{tabular}

* Para todos los casos, pro,0001 

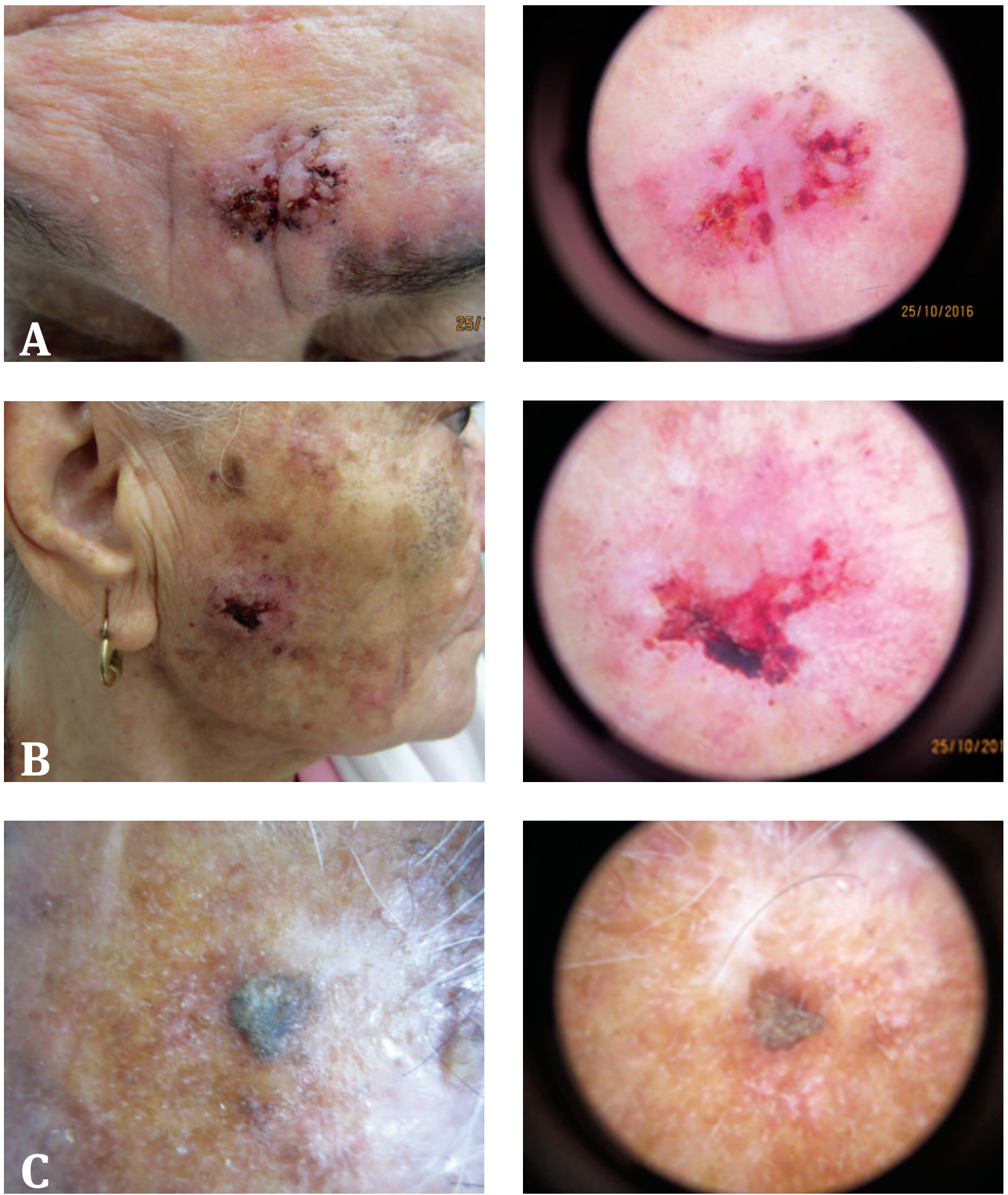

Figura 1. Ejemplos de lesiones con diagnósticos discordantes entre los distintos evaluadores. A) Fotografía clínica y dermatoscópica de la lesión $\mathrm{N}^{\circ}$ 32, diagnosticada por los evaluadores presenciales y uno de los teleevaluadores como carcinoma basocelular, y por los otros dos teleevaluadores, como carcinoma escamocelular. B) Lesión $\mathrm{N}^{\circ} 33$ diagnosticada como carcinoma basocelular por los dos evaluadores presenciales y uno de los teleevaluadores, y como carcinoma escamocelular, por los otros dos teleevaluadores. C) Lesión $\mathrm{N}^{\circ} 41$ diagnosticada como queratosis actínica por los dos evaluadores presenciales y, como queratosis seborreica, por los tres teleevaluadores. 

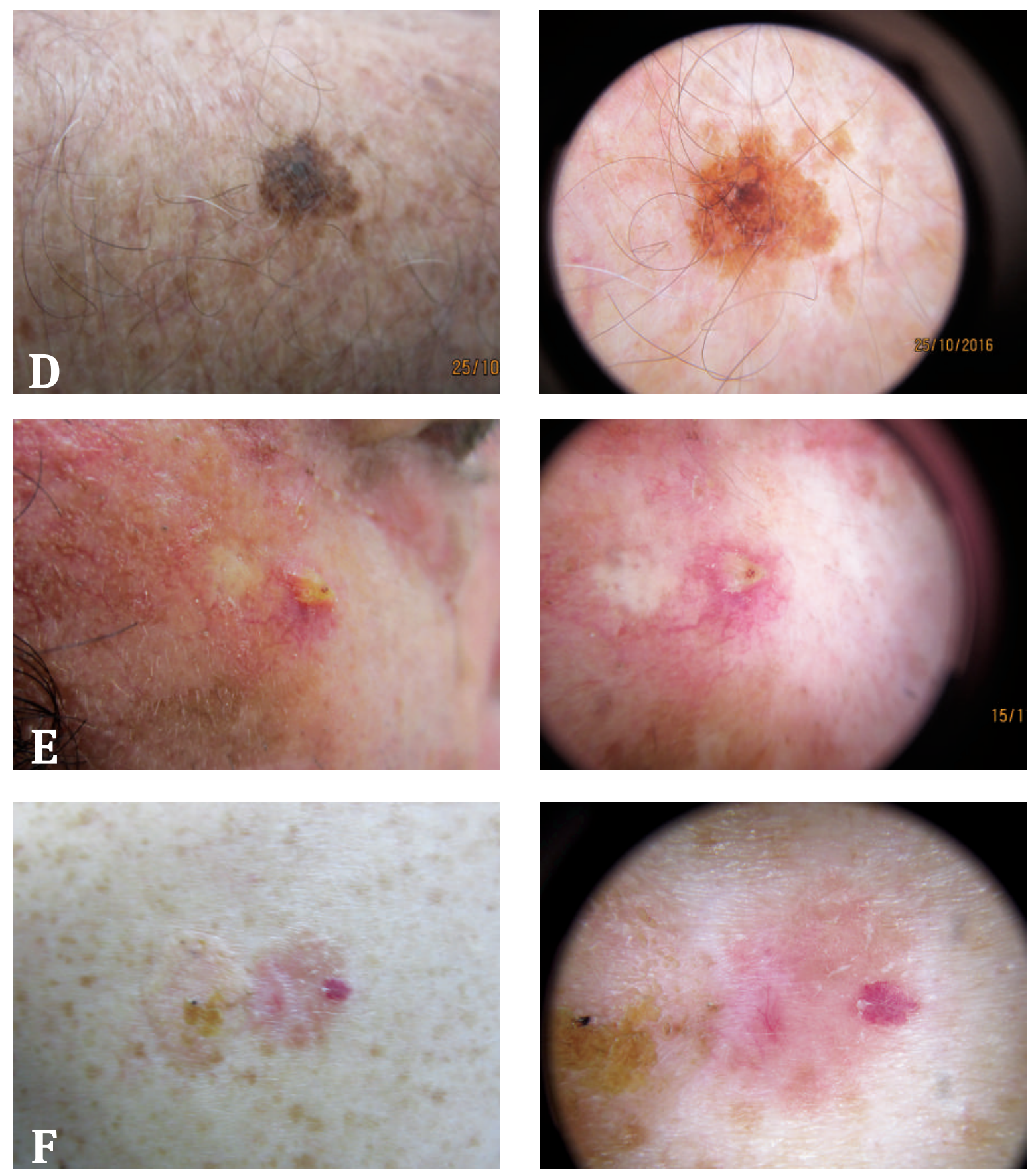

Figura 1. Ejemplos de lesiones con diagnósticos discordantes entre los distintos evaluadores. D) Lesión $\mathrm{N}^{\circ} 45$ diagnosticada como melanoma in situ por uno de los evaluadores presenciales y uno de los teleevaluadores, y como lentigo solar, por el resto de dermatólogos. E) Lesión $\mathrm{N}^{\circ} 48$ diagnosticada como queratosis actínica por los dos evaluadores presenciales y uno de los teleevaluadores, y como carcinoma escamocelular, por dos de los teleevaluadores. F) Lesión $\mathrm{N}^{\circ} 59$ diagnosticada como carcinoma basocelular por los dos evaluadores presenciales y dos de los teleevaludores, y como melanoma amelanótico, por uno de los teleevaluadores. G) Lesión $\mathrm{N}^{\circ} 64$ diagnosticada como léntigo solar por uno de los evaluadores presenciales, melanoma in situ por otro de los evaluadores presenciales, melanoma in situ por uno de los teleevaluadores, queratosis seborreica por otro de los teleevaludores, y queratosis actínica pigmentada por otro de los teleevaluadores. 


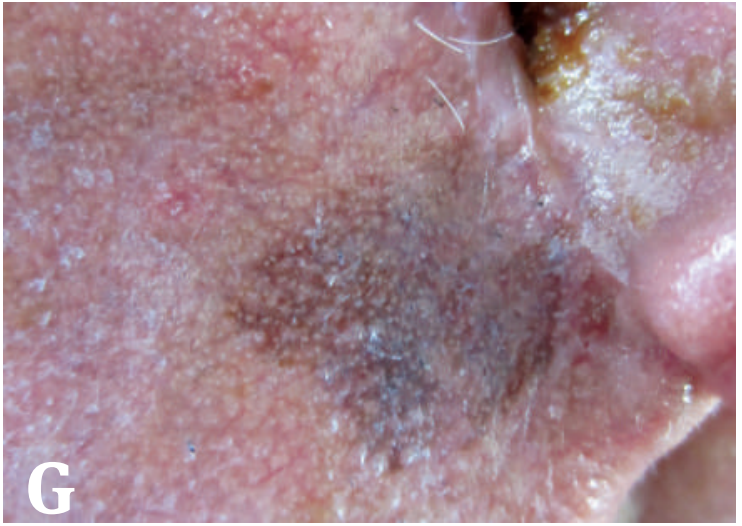

\section{DISCUSIÓN}

Se han hecho estudios de similar metodología en el mundo ${ }^{(4,5,11,12)}$. La concordancia reportada en la literatura científica entre los diagnósticos por teledermatoscopia y aquellos hechos mediante examen clínico presencial, varía sustancialmente ${ }^{(6)}$, con coeficientes kappa de 0,62 ${ }^{(13)}$ a 0,2 ${ }^{(14)}$, de 0,32 a 0,62 ${ }^{(15)}$, y de 0,41 a $0,63^{(16)}$ para el diagnóstico de lesiones tumorales.

Las concordancias varían según los diagnósticos, si son lesiones pigmentadas versus no pigmentadas ${ }^{(15)}$, si son lesiones que los examinadores consideraron de difícil de diagnóstico ${ }^{(11)}$, o si se hizo análisis por diagnóstico nosológico o por subgrupos ${ }^{(4,15)}$. Por ejemplo, cuando se agrupan el diagnóstico principal y el diagnóstico diferencial ${ }^{(15)}$ o cuando se agrupan los diagnósticos dentro del mismo espectro ${ }^{(4)}$; por ejemplo, queratosis actínicas y carcinoma escamocelular in situ o lesiones malignas y benignas (kappa=0,50), melanocíticas o no melanocíticas (kappa: de 0,77 a 0,82) ${ }^{(17)}$; en estos casos el rendimiento diagnóstico fue mayor. Entre esas categorías, la de menor índice kappa fue la de lesiones melanocíticas malignas.

Esto concuerda con un estudio realizado en Colombia, en el que la relación entre la clínica y la histopatología fue menor para las lesiones clínicamente diagnosticadas como melanoma que para aquellas clasificadas como carcinoma basocelular, escamocelular, nevos o lesiones no malignas ${ }^{(18)}$. Se evidenció la mayor dificultad en la clínica como en la telemedicina, para el diagnóstico de melanoma.

La mayoría de los estudios de diagnóstico presencial versus telediagnóstico, presentan concordancias aceptables ${ }^{(3,19)}$. Una investigación adelantada en Colombia demostró concordancias aceptables, las cuales eran superiores para la enfermedad tumoral dermatológica

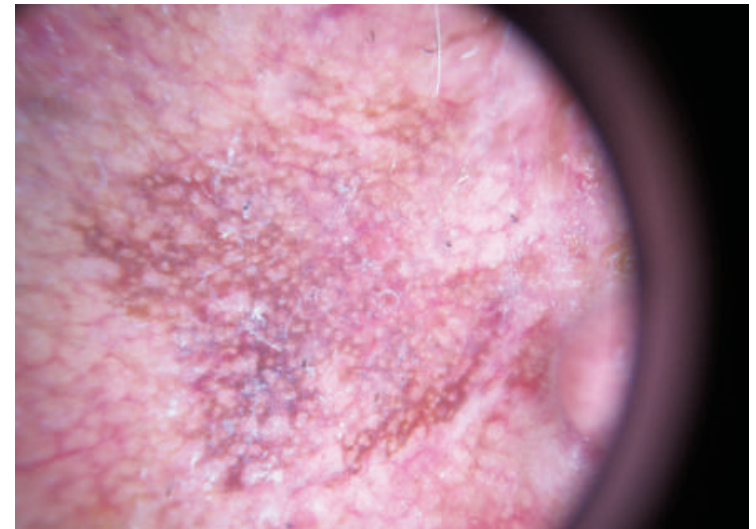

comparada con otras enfermedades ${ }^{(20)}$.

Las concordancias para la recomendación terapéutica entre evaluadores presenciales y teleevaluadores, se han reportado en índices kappa de 0,57 ${ }^{(13)}$, y de 0,19 a $0,41^{(15)}$. También, se ha hecho análisis de subgrupo y se ha encontrado que, para las lesiones no pigmen$\operatorname{tadas}^{(15)}$, el acuerdo es mayor que para las lesiones pigmentadas.

Al revisar lo descrito en la literatura científica, en nuestro estudio, el índice kappa para la clasificación de las lesiones y la recomendación terapéutica tuvo un excelente desempeño. Se han reportado índices kappa casi perfectos entre la evaluación teledermatoscópica y la evaluación presencial, cuando los teleevaluadores eran dermatólogos expertos en dermatoscopia ${ }^{(21)}$. Incluso, se ha descrito un mejor rendimiento diagnóstico por teledermatoscopia que mediante la evaluación presencial, usando como prueba de referencia la histopatología cuando los teleevaluadores tenían mayor experiencia ${ }^{(5,7)}$. En este estudio, hubo mayor concordancia entre el diagnóstico por imágenes de uno de los teleevaluadores -el de mayor experiencia en dermatoscopia- y el diagnóstico histopatológico, lo cual pone de manifiesto que el entrenamiento en dermatoscopia juega un papel importante en la precisión diagnóstica. Se han reportado múltiples experiencias ${ }^{(22-25)}$, incluida una revisión sistemática de la literatura ${ }^{(6)}$, en las cuales la atención por telemedicina para triage de lesiones tumorales disminuye el tiempo de espera y aumenta la oportunidad de atención en los casos que se consideran más urgentes.

En nuestro medio, donde muchos pacientes consultan por melanomas muy avanzados, que podrían haber sido diagnosticados de manera más temprana utilizando la modalidad de atención de telemedicina para triage de lesiones en áreas remotas sin acceso al 
especialista, podría ser una estrategia valiosa que se debería considerar. Incluso, en algunos estudios se ha demostrado, por medio de la teledermatología, el diagnóstico de melanomas con menor Breslow y mayor detección de carcinomas basocelulares ${ }^{(26)}$.

Se deben considerar también las desventajas de este modelo de atención. En la consulta dermatológica presencial, se pueden encontrar incidentalmente lesiones sospechosas por las que el paciente no consulta y que no fueron consideradas de importancia por el médico remisor y, por ende, no reciben atención ni tratamiento (27).

Los aspectos médico-legales y de responsabilidad médica por errores diagnósticos y aspectos éticos, por la modificación de la relación médico-paciente también son asuntos que aún no están resueltos. Falta superar barreras tecnológicas, como plataformas confiables con historias clínicas electrónicas adecuadas, que estén disponibles en lugares remotos para la atención por telemedicina y el cobro por la atención a distancia aún no está regulado ${ }^{(26)}$.

La calidad de las fotografías también puede ser una fuente de error si se encuentran borrosas, no se enfocan las estructuras relevantes, etc. Sin embargo, el cáncer de piel amerita una atención priorizada y los pacientes con cáncer de piel no tendrían por qué tener los mismos tiempos de espera para la atención especializada que aquellos con verrugas virales o queratosis seborreicas ${ }^{(26)}$.

Se deben implementar estrategias que permitan un diagnóstico más temprano si queremos tener un impacto en los desenlaces de nuestros pacientes. La teledermatología se considera costo-efectiva y se han reportado altos índices de satisfacción por los pacientes como un método de tamización para la detección temprana del cáncer de piel ${ }^{(28,29)}$.

Con este artículo se aporta la información con la que contamos actualmente para apoyar la modalidad de atención de telemedicina para el triage de lesiones tumorales en piel, mas no para remplazar el examen físico presencial por los sesgos de selección de lesiones por las que el paciente consulta; sin embargo, permite a los pacientes recibir atención especializada oportuna por lesiones que le preocupan. Con los hallazgos, es claro que es muy valioso como método clasificatorio para priorizar la atención de los pacientes, pero para hacer el diagnóstico preciso, no tiene tan buenos resultados. El melanoma continúa siendo una de las lesiones más difíciles de diagnosticar tanto por clínica como por telemedicina, siendo los de menor índice kappa para concordancia entre el diagnóstico presencial y el telediagnóstico, y menor correlación clínico- patológica en comparación con el resto del espectro analizado de lesiones tumorales.

También, apoya la 'evidencia' ya disponible de que, a mayor experiencia en dermatoscopia, mejor el rendimiento diagnóstico y, posiblemente, esto tenga implicaciones como quiénes son los dermatólogos idóneos para la práctica de la teledermatología o que los expertos tengan un papel importante en la teledermatología como herramienta para segundas opiniones o para consultoría en casos difíciles.

Es importante resaltar que todos los dermatólogos evaluadores participantes en este estudio, se dedican la mayor parte de su tiempo a la evaluación de lesiones tumorales, y que con los dermatólogos que no dedican todo su tiempo al diagnóstico y el tratamiento de lesiones tumorales los resultados podrían ser diferentes.

\section{REFERENCIAS}

1. Kanthraj GR. Newer insights in teledermatology practice. Indian J Dermatol Venereol Leprol. 2011;77:276-87.

2. Mehrtens SH, Halpern SM. Changing use and attitudes towards teledermatology in the UK over 10 years: Results of the 2016 National Survey. Br J Dermatol. Br J Dermatol. 2018;178:286-8.

3. Trettel A, Eissing L, Augustin M. Telemedicine in dermatology: Findings and experiences worldwide - a systematic literature review. J Eur Acad Dermatol Venereol. 2018;32:215-24.

4. Tan E, Oakley A, Soyer HP, Haskett M, Marghoob $\mathrm{A}$, Jameson $\mathrm{M}$, et al. Interobserver variability of teledermoscopy: An international study. Br J Dermatol. 2010;163:1276-81.

5. Tan E, Yung A, Jameson M, Oakley A, Rademaker M. Successful triage of patients referred to a skin lesion clinic using teledermoscopy (IMAGE IT trial): Teledermoscopy as triage for skin lesions. Br J Dermatol. 2009;162:803-11.

6. Bruce AF, Mallow JA, Theeke LA. The use of teledermoscopy in the accurate identification of cancerous skin lesions in the adult population: A systematic review. J Telemed Telecare. 2018;24:75-83.

7. Piccolo D, Smolle J, Argenziano G, Wolf IH, Braun $\mathrm{R}$, Cerroni L, et al. Teledermoscopy--results of a multicentre study on 43 pigmented skin lesions. J Telemed Telecare. 2000;6:132-7.

8. Warshaw EM, Gravely AA, Nelson DB. Accuracy of teledermatology/teledermoscopy and clinic-based dermatology for specific categories of skin neoplasms. J Am Acad Dermatol. 2010;63:348-52. 
9. Ferrándiz L, Ojeda-Vila T, Corrales A, Martín-Gutiérrez FJ, Ruiz-de-Casas A, Galdeano R, et al. Internet-based skin cancer screening using clinical images alone or in conjunction with dermoscopic images: A randomized teledermoscopy trial. J Am Acad Dermatol. 2017;76:676-82.

10. Landis JR, Koch GG. The measurement of observer agreement for categorical data. Biometrics. 1977;33:159-74.

11. Piccolo D, Smolle J, Wolf IH, Peris K, Hofmann-Wellenhof R, Dell'Eva G, et al. Face-to-face diagnosis vs telediagnosis of pigmented skin tumors: A teledermoscopic study. Arch Dermatol. 1999;135:146771.

12. Piccolo D, Soyer HP, Chimenti S, Argenziano G, Bartenjev I, Hofmann-Wellenhof $\mathrm{R}$, et al. Diagnosis and categorization of acral melanocytic lesions using teledermoscopy. J Telemed Telecare. 2004;10:346-50.

13. Lamel SA, Haldeman KM, Ely H, Kovarik CL, Pak $\mathrm{H}$, Armstrong AW. Application of mobile teledermatology for skin cancer screening. J Am Acad Dermatol. 2012;67:576-81.

14. Markun S, Scherz N, Rosemann T, Tandjung R, Braun RP. Mobile teledermatology for skin cancer screening: A diagnostic accuracy study. Medicine (Baltimore). 2017;96:e6278.

15. Warshaw EM, Gravely AA, Nelson DB. Reliability of store and forward teledermatology for skin neoplasms. J Am Acad Dermatol. 2015;72:426-35.

16. Finnane A, Dallest K, Janda M, Soyer HP. Teledermatology for the diagnosis and management of skin cancer: A systematic review. JAMA Dermatol. 2017;153:319-27.

17. Börve A, Terstappen K, Sandberg C, Paoli J. Mobile teledermoscopy-there's an app for that! Dermatol Pract Concept. 2013;3:41-8.

18. Velásquez MM, Zuluaga A. Primera jornada de detección precoz del cáncer de piel, Asocolderma, 2011, reporte de la experiencia en Medellín, Colombia. Rev Asoc Colomb Dermatol. 2012;20:13546.

19. Walocko FM, Tejasvi T. Teledermatology applications in skin cancer diagnosis. Dermatol Clin. 2017;35:559-63.

20. Mora O, Olmos E, Rochel C, Torres M, Rodríguez N. Acuerdo entre el examen dermatológico directo y teledermatología asincrónica. Prueba piloto, Hospital de San José, Bogotá, D.C., Colombia. Revista Repertorio de Medicina y Cirugía. 2012;21:122-5.

21. Arzberger E, Curiel-Lewandrowski C, Blum A, Chubisov D, Oakley A, Rademaker M, et al. Tele- dermoscopy in high-risk melanoma patients: A comparative study of face-to-face and teledermatology visits. Acta Derm Venereol. 2016;96:779-83.

22. Hue L, Makhloufi S, Sall N'Diaye P, BlanchetBardon C, Sulimovic L, Pomykala F, et al. Realtime mobile teledermoscopy for skin cancer screening targeting an agricultural population: An experiment on 289 patients in France. J Eur Acad Dermatol Venereol. 2016;30:20-4.

23. Kahn E, Sossong S, Goh A, Carpenter D, Goldstein S. Evaluation of skin cancer in Northern California Kaiser Permanente's store-and-forward teledermatology referral program. Telemed J. 2013;19:780-5.

24. Lim D, Oakley AMM, Rademaker M. Better, sooner, more convenient: A successful teledermoscopy service. Australas J Dermatol. 2012;53:22-5.

25. Börve A, Gyllencreutz J, Terstappen K, Backman E, Aldenbratt A, Danielsson M, et al. Smartphone teledermoscopy referrals: A novel process for improved triage of skin cancer patients. Acta Derm Venereol. 2015;95:186-90.

26. Moreno-Ramírez D, Ferrándiz L. A 10-year history of teledermatology for skin cancer management. JAMA Dermatol. 2015;51:1289-90.

27. Viola KV, Tolpinrud WL, Gross CP, Kirsner RS, Imaeda S, Federman DG. Outcomes of referral to dermatology for suspicious lesions: Implications for teledermatology. Arch Dermatol. 2011;147:55660.

28. Livingstone J, Solomon J. An assessment of the cost-effectiveness, safety of referral and patient satisfaction of a general practice teledermatology service. Lond J Prim Care. 2015;7:31-5.

29. Spinks J, Janda M, Soyer HP, Whitty JA. Consumer preferences for teledermoscopy screening to detect melanoma early. J Telemed Telecare. 2016;22:39-46. 